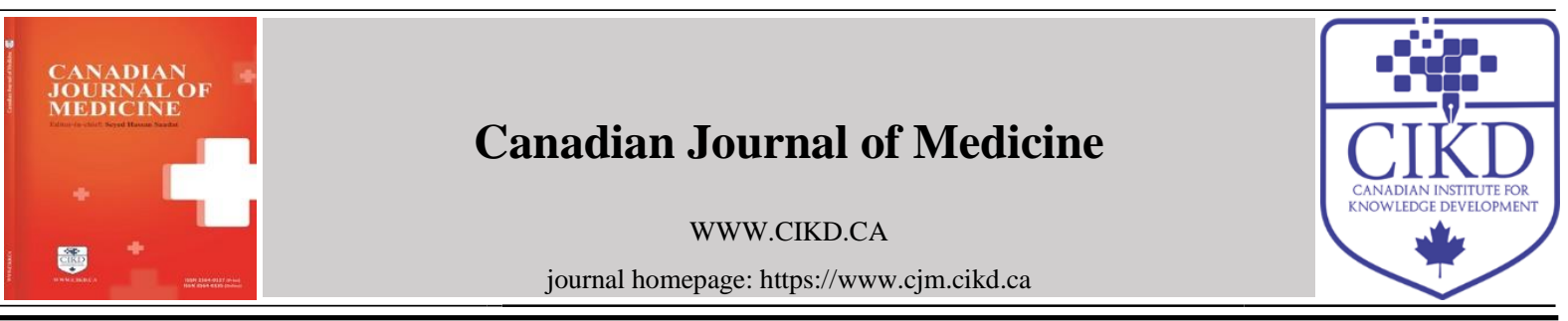

\title{
Autophagy and Coronavirus Interaction: Its Significance for COVID-19 Treatment
}

\author{
Pallab Chakraborty \\ University of Calcutta, India
}

Keywords:

Autophagy, Coronavirus

interaction, COVID-19

\section{Received}

13 September 2021

Received in revised form

26 September 2021

Accepted

26 September 2021

*Correspondence:

pallabchakraborty59@gmail.com
Dear Editor,

In December 2019, severe acute respiratory syndrome coronavirus 2 (SARS-CoV-2) emerged in Wuhan, Hubei province, China $[1,2]$. According to the literature, the coronavirus was found to interact with autophagy pathways [3-6]. The autophagy process (recycling pathway) is very important for the degradation of cytosolic compounds and the creation of building blocks in cells [7]. During starvation conditions, this process can be activated. It delivers the cytosolic components, including the damaged cellular organelles and mis-folded proteins, into lysosomes by establishing autophagosomes, where the degradation takes place [3,8]. It is reported to have a paradoxical role in protecting cells from viral and bacterial infections, depending on the types of pathogens and the host cells [3, 9]. Commonly known processes are xenophagy (viral particle degraded) and virophagy (degradation of neosynthesized components from virus), and innate- adaptive immune induction, by which autophagy contributes to the antiviral response $[8,10,11]$.

CCIKD Publishing

Dear Editor,

In December 2019, severe acute respiratory syndrome coronavirus 2 (SARS-CoV-2) emerged in Wuhan, Hubei province, China $[1,2]$. According to the literature, the coronavirus was found to interact with autophagy pathways [3-6]. The autophagy process (recycling pathway) is very important for the degradation of cytosolic compounds and the creation of building blocks in cells [7]. During starvation conditions, this process can be activated. It delivers the cytosolic components, including the damaged cellular organelles and mis-folded proteins, into lysosomes by establishing autophagosomes, where the degradation takes place $[3,8]$. It is reported to have a paradoxical role in protecting cells from viral and bacterial infections, depending on the types of pathogens and the host cells $[3,9]$. Commonly known processes are 
xenophagy (viral particle degraded) and virophagy (degradation of neosynthesized components from virus), and innate- adaptive immune induction, by which autophagy contributes to the antiviral response $[8,10,11]$. SARS CoV infection is capable of increasing the autophagosome formation, and it is assumed to activate autophagic flux by decreasing the angiotensin-converting enzyme 2 (ACE2) expression and leads to activation of the AMPprotein activated kinase (AMPK) or inhibition of the mammalian target of rapamycin (mTOR) [3]. Cottam et al. reported that the NSP6 protein of SARS could be capable of activating autophagy by increasing the level of phosphatidylinositol 3-phosphate (PtdIns3P), recruiting the double-FYVE-containing protein 1 (DFCP1) for the generation of autophagosomes from the ER [12]. Furthermore, the SARS-CoV open reading frame (ORF) $8 \mathrm{~b}$ aggregates were reported to be capable of triggering cellular stress, leading to autophagic efflux [3]. On the other hand, similar to mouse hepatitis virus (MHV) infection, doublemembrane vesicles (DMVs) creation has been observed in SARS-CoV-infected cells [3]. This double-membrane vesicle is related to the autophagosome, which fuses with the lysosome, and is associated with the canonical autophagy pathway [4]. These lysosomal fusion and acidification are vital in some coronaviruses for the genome release process into the host cytoplasm [4, 13]. In MHV, the replication is also reported to decrease up to 1000 fold in embryonic stem cells without autophagy-related protein 5 (ATG5-/-) compared to wild types with ATG5 expression ability [3]. On the other hand, Middle East respiratory syndrome coronavirus (MERS-CoV) was found to decrease the BECN1 protein because it is capable of activating the S-phase kinase-associated protein 2 (SKP2) [4], in turn, results in BECN1 protein degradation, leading to prevention of autophagosome-lysosome fusion $[3,4]$ as a possible protective strategy (to stop viral replication complex damage located on DMVs) [4]. It has been reported that ATG5 gene deletion in Vero B4 cells assisted replication 6-fold and helped in viral particle formation of MERS-CoV. By inhibiting SKP2, it was found that this elevation of BECN1 performance and autophagy can reduce MERS-CoV replication up to 28,000 - fold $[3,14]$.

It has been found that ORF3a as an accessory protein could initiate incomplete cellular autophagy for double-membrane autophagosome vesicles formation, where it is vital for efficient SARS-CoV-2 replication [15]. It has also been reported that SARS-CoV-2 replication efficiency is reduced by using the genetic knockout process for autophagic genes (Atg3 or Atg5 knockout) [15]. Another study supports that autophagosome formation in COVID-19 infection may be important for the viral replication where knockout of Vps15 or Beclin 1 was reported to be fully associated with blocked SARS-CoV-2 replication in Huh7.5.1 cells [16]. Furthermore, another study by Bouhaddou et al. demonstrated that the p38/MAPK signaling pathway was activated in SARS-CoV-2 infected Vero E6 cells, while PIK3CA/AKT was found to be inhibited, resulting in induction of autophagy [3,17]. Furthermore, Gordon et al. showed that the $\mathrm{N}$ and the ORF8 proteins of the COVID 19 virus led to MTORC1 inhibition, which is associated with mTOR signaling, including growth and stress, etc. [3].

A growing body of evidence indicates that both SARS CoV and MERS CoV virus preferred autophagy induction for their existence but may not prefer autophagolysosome formation. Thus, inhibition of autophagy or autophagy-related genes could negatively affect 
virus replication and positively affect treatment. Although the complete understanding of autophagy in MERS-CoV infection and SARS-CoV 2 is still unclear, it is essential to figure out the exact role of autophagy and its signaling before any helpful and constructive feedback. Literature also suggests that SARS-CoV 2 could interact with the autophagy process for its replication, but more evidence and further holistic researches in terms of autophagy mechanism are required. Rayner et al. showed that AR12 (OSU-03012) suppressed infection by autophagic flux improvement in SARS CoV-2 infected Vero cells [18]. Moreover, autophagy is known to adjust the inflammatory response (control of over-response) and divers' antiviral immune response, but inflammation was found to be uncontrolled in COVID19 patients. Therefore, activation of the autophagy pathway or any autophagy-related genes could be a potential approach in such conditions to maintain homeostasis [8]. Another study about autophagy-related proteins reported that decreasing their level (LC3 in patients of any age and SQSTM1 in patients up to 50 years old) is associated with moderate-to-severe COVID-19 [19, 20].

Several FDA-approved drugs are interconnected to have autophagy modulation property and antiviral activity against human COVID-19, reviewed in the literature [6, 20, 21]. Finally, it is very important to know the mechanism behind autophagy and COVID-19 for better treatment. For now, the use of approved drugs could be beneficial for rapid patient access to COVID-19 treatments. In the future, detailed study of how the virus regulates the pathway, understanding the cell biology of COVID-19 infection, and clinical trials with drugs to target autophagy and associated signaling proteins with other therapies can be of great importance.

\section{References}

[1] Li H, Liu S, Yu X, Tang S, Tang C. Coronavirus disease 2019 (COVID-19): current status and future perspectives. International Journal of Antimicrobial Agents. 2020; 55(5): p105951.

[2] COVID-19 Coronavirus Pandemic live update. Available from: https://www.worldometers.info/coronavirus/ [Accessed 23 May 2021] [homepage on the Internet]. n.d.

[3] Zhao Z, Lu K, Mao B, Liu S, Trilling M, Huang A, Lu M, Lin Y. The interplay between emerging human coronavirus infections and autophagy. Emerging Microbes \& Infections. 2021;10(1):196-205.

[4] Miller K, McGrath ME, Hu Z, Ariannejad S, Weston S, Frieman M, Jackson WT. Coronavirus interactions with the cellular autophagy machinery. Autophagy. 2020;16(12): 2131-9.

[5] Delorme-Axford E, Klionsky DJ. Highlights in the fight against COVID-19: does autophagy play a role in SARS-CoV2 infection?. Autophagy. 2020;16(12):2123-2127.

[6] Bello-perez M, Sola I, Novoa B, Klionsky DJ, Falco A. Canonical and Noncanonical Autophagy as Potential Targets for COVID-19 Cells. 2020;9(7):1619.

[7] Mizushima N. Autophagy: process and function. Genes \& development. 2007;21(22):2861-73.

[8] García-Pérez BE, González-Rojas JA, Salazar MI, Torres-Torres C, Castrejón-Jiménez NS. Taming the Autophagy as a Strategy for Treating COVID-19. Cells. 2020;9(12):2679.

[9] Shibutani ST, Saitoh T, Nowag H, Münz C, Yoshimori T. Autophagy and autophagy-related proteins in the immune system. Nature Immunology. 2015;16(10):1014-24.

[10] Orvedahl A, Sumpter R, Xiao G, Ng A, Zou Z, Tang Y, Narimatsu M, Gilpin C, Sun Q, Roth M, Forst CV. Imagebased genome-wide siRNA screen identifies selective autophagy factors. Nature. 2011;480(7375):113-7.

[11] Galluzzi L, Baehrecke EH, Ballabio A, Boya P, Bravo-San Pedro JM, Cecconi F, Choi AM, Chu CT, Codogno P, Colombo MI, Cuervo AM. Molecular definitions of autophagy and related processes. The EMBOJournal. 2017;36(13):1811-36. 
[12] Cottam EM, Maier HJ, Manifava M, Vaux LC, Chandra-Schoenfelder P, Gerner W, Britton P, Ktistakis NT, Wileman T. Coronavirus nsp6 proteins generate autophagosomes from the endoplasmic reticulum via an omegasome intermediate. Autophagy. 2011;7(11):1335-47.

[13] Milewska A, Nowak P, Owczarek K, Szczepanski A, Zarebski M, Hoang A, Berniak K, Wojarski J, Zeglen S, Baster Z, Rajfur Z. Entry of human coronavirus NL63 into the cell. Journal of Virology. 2018;92(3):e01933-17.

[14] Gassen NC, Niemeyer D, Muth D, Corman VM, Martinelli S, Gassen A, Hafner K, Papies J, Mösbauer K, Zellner A, Zannas AS. SKP2 attenuates autophagy through Beclin1-ubiquitination and its inhibition reduces MERS-Coronavirus infection. Nature Communications. 2019;10(1):1-6.

[15] Qu Y, Wang X, Zhu Y, Wang W, Wang Y, Hu G, Liu C, Li J, Ren S, Xiao MZ, Liu Z. ORF3a-Mediated Incomplete Autophagy Facilitates Severe Acute Respiratory Syndrome Coronavirus-2 Replication. Frontiers in Cell and Developmental Biology. 2021;9:2012.

[16] Wang R, Simoneau CR, Kulsuptrakul J, Bouhaddou M, Travisano KA, Hayashi JM, Carlson-Stevermer J, Zengel JR, Richards CM, Fozouni P, Oki J. Genetic screens identify host factors for SARS-CoV-2 and common cold coronaviruses. Cell. 2021;184(1):106-19.

[17] Bouhaddou M, Memon D, Meyer B, White KM, Rezelj VV, Marrero MC, Polacco BJ, Melnyk JE, Ulferts S, Kaake RM, Batra J. The global phosphorylation landscape of SARS-CoV-2 infection. Cell. 2020 ;182(3):685-712.

[18] Rayner JO, Roberts RA, Kim J, Poklepovic A, Roberts JL, Booth L, Dent P. AR12 (OSU-03012) suppresses GRP78 expression and inhibits SARS-CoV-2 replication. Biochemical Pharmacology. 2020;182:114227.

[19] Fang S, Zhang L, Liu Y, Xu W, Wu W, Huang Z, Wang X, Liu H, Sun Y, Zhang R, Peng B. Lysosome activation in peripheral blood mononuclear cells and prognostic significance of circulating LC3B in COVID-19. Briefings in bioinformatics. 2021;22(2):1466-75.

[20] Shroff A, Nazarko TY. The Molecular Interplay between Human Coronaviruses and Autophagy. Cells. 2021;10(8):2022.

[21] Pereira GJ, Leão AH, Erustes AG, Morais IB, Vrechi TA, Zamarioli LD, Pereira CA, Marchioro LD, Sperandio LP, Lins ÍV, Piacentini M. Pharmacological Modulators of Autophagy as a Potential Strategy for the Treatment of COVID19. International Journal of Molecular Sciences. 2021;22(8):e4067.

\title{
Author's contribution
}

This letter to the editor was prepared and critically revised by the author. However, the author makes no representations that the data available in the referenced papers are free from error.

\section{Acknowledgements}

Not applicable.

\section{Disclosure Statement}

No potential conflict of interest was reported by the authors.

\section{Funding Acknowledgements}

\author{
Not applicable.
}

